\title{
Doença de Alzheimer: a experiência única de cuidadores familiares
}

\author{
Emanuela Bezerra Torres Mattos ${ }^{\text {a* }}$ () \\ Maria Julia Kovács ${ }^{\mathbf{b}}$ (i)
}

\author{
a Instituto Saúde e Sociedade da Unifesp, Departamento Saúde Educação e Sociedade. São Paulo, SP, Brasil \\ ${ }^{b}$ Instituto de Psicologia da USP, Departamento de Psicologia da Aprendizagem, \\ do Desenvolvimento e da Personalidade. São Paulo, SP, Brasil
}

\begin{abstract}
Resumo: A demência é uma síndrome de curso lento, progressivo e de natureza crônica, sendo o subtipo doença de Alzheimer (DA) a mais comum. Muitos estudos são realizados a partir das demandas da pessoa com demência, porém na perspectiva do cuidador eles são escassos. A pesquisa qualitativa teve como objetivo conhecer aspectos singulares da experiência do cuidar na perspectiva de cuidadoras familiares de idosos com DA, a partir de abordagem qualitativa. Foi utilizado o método fenomenológico proposto por Giorgi e Sousa. Participaram nove cuidadoras familiares (quatro esposas e cinco filhas) que cuidam de seus familiares. Esta pesquisa acompanhou o cuidar na fase inicial, moderada, avançada, em diferentes estágios da doença e no pós-óbito. A partir da questão central: "Como é para você a experiência de acompanhar seu familiar com a doença de Alzheimer?", emergiram treze unidades temáticas que foram associadas às fases da doença. Os resultados apontaram as necessidades dos cuidadores, que vão desde o diagnóstico em fases iniciais até a criação de espaço para escuta e acolhimento diante das perdas graduais vivenciadas ao longo do processo de cuidar. Assim, é urgente o investimento em formação de profissionais em todas as áreas envolvidas no cuidar para promover qualidade de vida e bem-estar aos cuidadores. bem como a necessidade de equipes interdisciplinares para a experiência singular do cuidado em demência.
\end{abstract}

Palavras-chave: demência, doença de Alzheimer, cuidador familiar.

\section{Introdução}

\section{Demência da doença de Alzheimer}

Em todo o mundo, a maior expectativa de vida favorece o aumento do número de pessoas com demência. Recentemente, a demência passou a ser denominada pelo Diagnostic and statistical manual of mental disorders (DSM-V), como Transtorno Neurocognitivo Maior (TNM) (American Psychiatric Association, 2014). Por ser uma entidade nosológica reconhecida mundialmente ainda como demência, optamos por manter essa expressão ao longo deste artigo. Em 2015, aproximadamente 46,8 milhões de pessoas foram diagnosticadas com demência, e em 2050 esse número poderá atingir 131,5 milhões (Alzheimer's Disease International, 2016).

A demência é uma síndrome causada por várias doenças de curso lento, progressivo, evolutivo e de natureza crônica, sendo que o subtipo doença de Alzheimer (DA) corresponde à maior parte dos casos diagnosticados (Burlá, 2015).

\footnotetext{
* Endereço para correspondência: emattos@unifesp.br
}

\section{Cuidador familiar}

Apesar do conceito de cuidador ser bastante discutido na literatura mundial, ainda não há consenso sobre sua definição, havendo discordâncias nos diversos estudos que envolvem essa temática.

O curso gradual, progressivo e irreversível da DA compromete não somente o paciente, mas também seus cuidadores e sua família, pois favorece a construção de uma cadeia de perdas simbólicas, definida pela literatura internacional como luto antecipatório (Farran, Keane-Hagerty, Salloway, Kupferer, \& Wilken, 1991; Holley, 2009; Sanders \& Corley; 2003).

\section{Luto antecipatório}

O termo luto antecipatório foi utilizado pela primeira vez por Lindemann, em 1944, quando publicou o artigo intitulado "The symptomatology and management of acute grief" (Fonseca, 2004). Esse termo é definido como o processo pelo qual famílias antecipam e tentam elaborar as perdas futuras, ainda não vinculadas à morte concreta, mas, sim, às perdas pessoais, da identidade e do que o paciente representava para a família (Rolland, 1998).

Rando (1986) propôs o modelo multidimensional de luto antecipatório que pode ser adequado às doenças 
crônicas degenerativas, dentre elas, a demência. Nesse contexto, o luto antecipatório na perspectiva do cuidador diante das perdas cognitivas de seu familiar implica o luto vivenciado pelo futuro desconhecido e pelas mudanças ocorridas no presente em relação ao passado vivido. Além disso, cabe ressaltar que o modelo postula que a forma de manifestação do luto antecipatório sofre influência de variáveis psicológicas, sociais e fisiológicas de quem desempenha esse cuidado.

Em contraste com a literatura sobre o luto experimentado imediatamente antes ou após a morte de um familiar, relativamente pouco se sabe a respeito do luto e das perdas relacionadas com a demência durante a trajetória do período de cuidado (Doka, 2004).

\section{Método}

Esta pesquisa teve como objetivo conhecer a experiência do cuidar na perspectiva de cuidadoras familiares de idosos com DA. Para isso, a abordagem qualitativa em pesquisa parece ser a mais adequada por considerar a análise da experiência dos atores envolvidos, além da subjetividade do pesquisador e do pesquisado, a abrangência do fenômeno investigado, situar e contextualizar os demais fenômenos, sejam eles sociais, culturais, econômicos, emocionais e/ou psicológicos (Morais, 2011).

O estudo foi submetido e aprovado pelo Comitê de Ética em Pesquisa com Seres Humanos da Universidade Federal de São Paulo, sob parecer $\mathrm{n}^{\mathrm{o}}$ 647.560. Após o aceite da instituição para o desenvolvimento da pesquisa, foram contatados, via telefone, todos os cuidadores familiares que participavam de projetos realizados na instituição. Nesse momento eles foram esclarecidos a respeito dos objetivos do estudo, dos procedimentos de coleta dos relatos e das questões éticas. Aqueles que mostraram interesse em colaborar agendaram uma conversa em dias, horários e locais predeterminados, de acordo com suas disponibilidades de tempo e demandas. Doze cuidadoras assinaram o Consentimento Livre e Esclarecido (TCLE), porém apenas nove participaram do estudo. Duas não conseguiram encaixar dias e horários para a entrevista, após três contatos telefônicos feitos pela pesquisadora. Uma preferiu não colaborar devido ao falecimento recente da mãe e à vivência de um processo doloroso de luto.

Apenas uma das nove cuidadoras optou por dar seu depoimento fora do ambiente domiciliar e justificou que não seria o ambiente propício, devido às demandas constantes de seu familiar com DA. As demais optaram pela entrevista no domicílio no horário de sono, descanso ou de atividades externas de seus familiares.

Participaram, assim, nove cuidadoras familiares (quatro esposas e cinco filhas) acompanhadas pelos grupos de suporte e apoio ao cuidador oferecidos no
Serviço Serviço de Atendimento ao Envelhecimento Cognitivo (SAEC) e pela Associação Brasileira de Alzheimer (ABRAz) Sub-regional Santos, localizados na Universidade Federal de São Paulo (Unifesp/Campus Baixada Santista).

Os critérios de inclusão foram: ser cuidadora principal de idoso com diagnóstico de DA e ser familiar, independentemente do grau de parentesco e do estágio da doença do idoso; no caso das entrevistas pós-óbito, ter sido cuidador por pelo menos seis meses no último ano de vida. Os critérios de exclusão eram: não ser familiar; ser familiar, mas receber algum tipo de remuneração para exercer essa função; ou estar há menos de seis meses nesse papel.

Das nove cuidadoras (C) familiares (F) entrevistadas, quatro eram esposas e cinco, filhas. A idade variou entre 51 a 80 anos. Sete moravam junto com o familiar, e duas das filhas não dormiam na mesma residência de seus familiares com DA. Para a descrição e análise dos relatos, elas foram enumeradas de $\mathrm{C} 1$ a C9, de acordo com a ordem cronológica das entrevistas. Duas delas vivenciaram no momento da entrevista a experiência de cuidar da pessoa com DA na fase leve, três na moderada, uma participou tanto na fase avançada quanto no pós-óbito e apenas uma no pós-óbito.

Foram respeitados os princípios éticos de sigilo, privacidade, confidencialidade, não identificação das colaboradoras, liberdade de participação e garantia de apoio psicológico, se necessário. Todos os depoimentos foram gravados e transcritos na íntegra a partir da entrevista aberta com uma questão norteadora: "Como é para você a experiência de acompanhar seu familiar com a demência da doença de Alzheimer?".

As unidades de significado (US) emergiram tendo como fundamento o método fenomenológico adaptado e proposto por Giorgi e Sousa (2010). Este é um método utilizado na abordagem qualitativa que propõe passos metodológicos para identificar unidades de significado que são: (1) estabelecer o sentido do todo; (2) determinar as partes e dividir as unidades de significado; e (3) transformar as US em expressões de caráter psicológico para alcançar as unidades temáticas que resultaram nesta pesquisa. Foram estabelecidas treze unidades de significados, obtidas a partir da análise dos nove depoimentos. Elas foram associadas às três fases da doença nas quais as cuidadoras vivenciaram a experiência do cuidar e do pós-óbito.

\section{Resultados}

Compreender a experiência de cuidadores familiares de idosos com demência ao longo do processo de cuidar permitiu desmistificar que o cuidado é essencialmente voltado à pessoa com demência. Faz-se necessário ampliar o olhar para o cuidador familiar, na medida em que as unidades de significados 
emergentes demonstram aspectos importantes a serem trabalhados no âmbito da saúde nos diferentes níveis de atenção, de maneira a garantir apoio e suporte desde a fase inicial até as demandas advindas com a morte do familiar. Para tal, é primordial o investimento em formação de profissionais em todas as áreas envolvidas para promover saúde, qualidade de vida e bem-estar aos cuidadores.

Quadro 1. Unidades de significados identificadas

\begin{tabular}{|c|c|c|c|}
\hline \multicolumn{3}{|c|}{ Unidades de significados } \\
\hline Fase inicial & Fase moderada & Fase avançada & $\begin{array}{c}\text { Temas transversais a todas } \\
\text { as fases e ao pós-óbito }\end{array}$ \\
\hline & - A ausência na presença; & \\
- A demora no diagnóstico; & "E se eu não estiver mais aqui, & & \\
- A busca por suporte & quem cuidará dele(a)?". & - Somente eu sei cuidar; \\
e orientação; & - A institucionalização como aliada ou vilã; & - Um caminhar \\
solitário & Luto antecipatório; \\
- Doença estigmatizada. & - A sobrecarga do cuidar; & Luão reconhecido \\
& socialmente. & \\
& - A doença de várias gerações; & & \\
\hline
\end{tabular}

\section{Fase inicial}

As três unidades de significados que compõem a fase inicial são:

\section{A demora no diagnóstico}

O diagnóstico precoce e preciso da demência, bem como a discussão do prognóstico e do manejo são cruciais e podem ter efeitos significativos a longo prazo para os pacientes, seus cuidadores e famílias. O diagnóstico de demência requer a coordenação eficaz entre serviços de cuidados primários e especializados. No entanto, em todo o mundo, famílias relatam atrasos no reconhecimento e diagnóstico das demências pela dificuldade no acesso a serviços de saúde, o que resulta em cuidados inconsistentes (Frank, 2017; Jamieson, Grealish, Brown, \& Draper, 2016; World Health Organization [WHO], 2012).

Nesse sentido, são paradigmáticos os depoimentos de C2 (esposa/62a): "Por que nenhum médico foi capaz de identificar?" e de C7(filha/58a): "O meu irmão começou a perceber que tinha alguma coisa diferente $e$, no começo, a gente até relutou". No aparecimento dos primeiros sintomas o médico teve dúvida ao dar o diagnóstico de Alzheimer para a mãe de $\mathrm{C} 7$, que aponta: "para eles (médicos) o Alzheimer ainda é uma coisa confusa, não é clara. Eles (os médicos) sabem pouco sobre isso".

Apesar de ser uma doença incurável, o tratamento farmacológico da DA em fases iniciais pode proporcionar a estabilização da doença por alguns anos e abrir possibilidades de encaminhamento de todos os envolvidos para outras especialidades, quando se fizer necessário, no intuito de acolher as demandas advindas com a doença, que tende a mudar de acordo com o perfil de cada pessoa e família (Frank, 2017).

\section{A busca por suporte e orientação}

A participação em grupos de apoio pode ser tão essencial para cuidadores, quanto os medicamentos são para pessoas com demência (Dizazzo-Miller, Samuel, Barnas, \& Welker, 2014). Estudo de follow-up por oito anos indicou que um programa intensivo de dez dias de treinamento para cuidadores impulsionado por acompanhamento e conferências telefônicas retardou a institucionalização de pessoas com demência (Brodaty, Green, Koschera, \& Koschera, 2003; Brodaty, Gresham, \& Luscombe, 1997). Outros estudos sugerem que os grupos de apoio voltados aos cuidadores não atendem adequadamente às suas expectativas (Eloniemi-Sulkava et al., 2009; Raivio, Laakkonen, \& Pitkälä, 2011).

Na realidade brasileira, a maior parte dos serviços disponíveis às pessoas com DA e/ou seus cuidadores/ famílias se dá em instituições privadas, em função da limitação na oferta de serviços públicos. Prorok, Horgan e Seitz (2013) identificaram que o caminho para acessar a assistência na atenção primária e nos serviços especializados foi longo.

As cuidadoras participantes desta pesquisa (C4/filha/62a, C5/filha/70a, C6/esposa/65a, C9/ filha/70a) demonstraram interesse na busca e acesso aos grupos de apoio psicoeducacional da Associação Brasileira de Alzheimer (ABRAz) como possibilidade de troca de experiências e orientação no manejo dos sintomas cognitivos e comportamentais. Sobre isso, C4 (filha/61a) fala:

Então, a gente quer uma ajuda para ver como é que a gente pode fazer para melhorar. Por isso que eu procurei o grupo de apoio. . . Você estando no grupo de apoio, os depoimentos são diferentes. São várias pessoas, então, cada um dá o seu depoimento de vida. Você vê que você também não está sozinha. 
Tem muitas famílias passando pelo mesmo problema que você. Há uma troca de experiências.

Estudo desenvolvido por Barnett et al. (2012) chama a atenção para os desafios envolvidos na prestação de cuidados às pessoas com demência e reconhece a necessidade de desenvolvimento de intervenções que alinhem cuidados de saúde afinados com as necessidades dos envolvidos ao longo do processo de conviver com as demências e as questões implicadas.

Num estudo da Universidade de Nova York, um projeto de intervenção com o cuidador identificou que aqueles que participaram de intervenções individuais, terapia familiar, grupos de apoio e aconselhamento, respeitando suas necessidades, relataram menos sintomas depressivos após a perda de seu familiar, quando comparados ao grupo-controle. Outros estudos chamam a atenção para o fato de que diferenças no tipo de intervenção podem explicar os padrões divergentes de resultados (Haley et al., 2008; Holland, Currier, $\&$ Gallagher-Thompson, 2009).

\section{Doença estigmatizada}

Doka (2004) recorda o clássico livro $A$ doença como metáfora, de Susan Sontag, no qual a autora argumenta que a doença não é apenas uma condição biológica, mas, sim, uma construção social e cultural. Cada sociedade atribui significados diferentes para a doença. Qual o significado atribuído à doença de Alzheimer? As experiências retratadas nas falas das cuidadoras chamam a atenção para uma doença que pode rotular as pessoas com a DA respectivamente de caducas, infantis, agressivas, doidas ou, mesmo, suicidas $(\mathrm{C1} /$ esposa/70a, C2/esposa/62a, C3/esposa/80a, C7/filha/58a).

Cada cultura apresenta significados diferentes para as doenças, mas a doença de Alzheimer em geral é associada a características pejorativas que tendem a excluir as pessoas de seu meio social. Na cultura americana, a demência geralmente é associada à insanidade e a comportamentos perigosos. Na China, a palavra demência é associada a "pessoas bobas". Outras culturas compreendem a doença como retribuição pelos erros da família, resultado de maldição, mau-olhado ou vontade de Deus (Doka, 2004).

Dessa forma, os significados culturalmente atribuídos se referem às doenças e também às pessoas que sofrem dela, seus cuidadores e família, e podem refletir a forma como a doença é percebida e tratada em cada país.

Consulta conduzida pela National Dementia Strategy na Inglaterra destacou o estigma em relação à demência como um dos fatores que colabora com as baixas taxas de diagnóstico da demência (WHO, 2012).

Dessa forma, é primordial que profissionais de saúde inseridos nos diversos níveis de atenção desenvolvam estratégias de percepção das necessidades do paciente e de suas famílias, e explorem o conhecimento sobre suas crenças e necessidades no intuito de eliminar lacunas que possam alimentar o estigma associado aos quadros demenciais.

\section{A fase moderada}

Sete unidades foram agregadas à fase intermediária da jornada, entre eles:

\section{A ausência na presença}

A percepção da presença física dos entes queridos, apesar de sua ausência psicológica, foi comum nas falas de C5, C6, C9.

Minha mãe sempre foi muito ativa, independente, esperta, inteligente, e você vê, assim, um processo de degeneração. É muito doloroso, sofrido. É um sofrimento constante. Tenho a sensação que tenho duas mães. Uma antes da doença, alegre e disposta, e a outra que quase não abre os olhos. Com o agravamento da doença, ela foi se afastando da gente, como se não estivesse presente ali. Isso dói muito, evidentemente. (C5/filha/70a)

\section{“E se eu não estiver mais aqui, quem cuidará dele (a)?"}

Inicialmente, esse questionamento pode sugerir o receio de que a pessoa com DA fique abandonada, caso o cuidador faleça, sem receber cuidados adequados no ambiente domiciliar e/ou familiar, ou que haja a necessidade de institucionalização, como aponta a cuidadora a seguir:

"Eu tenho medo de não ter forças, de não ter saúde
para cuidar dele. De eu adoecer e não ter como
cuidar. É a minha maior preocupação. No fim,
é provável que nós possamos ir e ele ficar. Se ele ficar,
quem é que vai cuidar? (C4/filha/61a)

Viver cotidianamente as limitações progressivas e degenerativas de um familiar com a demência da doença de Alzheimer pode suscitar medos, entre eles, o medo da incapacidade ou da morte. Kovács (2005) sugere que esta dificuldade reside no contexto sociocultural que, ao cultivar crenças que ressignificam a vida, rejeita a doença e nega a morte. C2, C4 e C6 são cuidadoras com mais de 60 anos, idade em que começa a surgir a consciência das perdas e da finitude da existência.

A rotina de sobrecarga física, emocional, econômica e social das cuidadoras familiares, associada à abdicação da vida anterior em que havia espaço para atividades profissionais, de cuidado com a saúde e lazer, pode favorecer a percepção de que elas estão mais susceptíveis ao adoecimento e à morte. Tal percepção 
pode gerar um círculo vicioso, em que o sofrimento psíquico e emocional leva a mais medo da morte.

\section{Somente eu sei cuidar}

O território da doença crônica pode ser comparado a um barco à deriva, quando se trata do cuidar de pessoas queridas. Para o familiar, mesmo sentindo que tem o controle, em alguns casos esse controle não é real ou efetivo (Gonzaga, 2012). Nesse sentido, C3 (esposa/80a) aponta:

Nunca faço nada agora. Não dá para desligar porque é uma coisa que está na gente. Enquanto eu tiver forças para carregar, ajudar a levar ele para cama, a subir, a descer, a trocar, eu farei. Eu sinto que meu marido se sente bem comigo.

Cruz e Hamdan (2008) afirmam que algumas pessoas na função de cuidador tendem a monopolizar a função, colocando-se na posição de serem os únicos a terem condições de realizar os melhores cuidados e abdicando de atividades que gerem satisfação pessoal, o que resulta no que os autores denominam de heroicamente estressados.

\section{A institucionalização como aliada ou vilã}

Muitas pessoas com demência tendem a ser cuidadas por equipamentos de saúde dedicados ao cuidado de longo prazo, pelas seguintes razões: a hospitalização da pessoa com demência devido à doença aguda, colapso ou morte do cuidador familiar (frequentemente idosos); sobrecarga do cuidador; preocupação com o bem-estar ou com a própria segurança do paciente (Small, Froggatt, $\&$ Downs, 2007).

De acordo com a definição da World Health Organization, as instituições de longa permanência para idosos (ILPI) estão inseridas na classificação the long term care por oferecerem cuidados médicos e/ou não médicos às pessoas com doenças ou incapacidades crônicas que necessitam de ajuda em suas atividades de vida diária por tempo prolongado (WHO, 2012). No Brasil, dados recentes apontam que menos de $1 \%$ da população idosa utiliza o serviço das instituições de longa permanência (ILPI). No retrato brasileiro, $65,2 \%$ das ILPI são filantrópicas, $28,2 \%$ são privadas e $96,6 \%$ públicas. Pesquisa nacional sobre instituições para idosos realizada em 2011 pelo Instituto de Pesquisa Econômica Aplicada (2011) chama a atenção para o crescimento da população que necessita de cuidados de longa duração, apesar da maioria das pessoas idosas manifestar o desejo de continuar a viver em suas próprias casas pelo maior tempo possível (WHO, 2012). O estigma social e cultural fortemente associado às casas geriátricas, casas de repouso, asilos, abrigos ou ILPI faz com que essas instituições sejam identificadas como lugar de abandono e desprezo (Silva, 2012). C3 (esposa/80a) fala: "Eu digo que seria a última coisa na minha vida seria internar ele numa clínica. A gente faz tudo, eu deixo tudo, mas fico com ele".

Esse aspecto pode ser paradoxal pois, para muitos idosos, a institucionalização pode significar o sentimento de pertencimento, amparo, proteção e segurança, nos casos em que o idoso se torna totalmente dependente, carente de renda, no caso de não ter constituído família ou viver situação familiar conflituosa (Instituto de Pesquisa Econômica Aplicada, 2011). C2 (esposa/62a), casada e sem filhos, encontrou com a institucionalização do marido aos fins de semana a possibilidade de ter um tempo para cuidar de si. Ela completa: "Me aliviou bastante. Cheguei à conclusão que o que importa é a gente viver bem" (C2/esposa/62a).

\section{A sobrecarga do cuidar}

Cuidar de pessoas com demência pode provocar nível significativo de sobrecarga, o que resulta em piores condições físicas e psicológicas (Bell, Araki, \& Neumann, 2001; Gallagher-Thompson \& Powers, 1997), levando à institucionalização prematura da pessoa com demência (Bédard, Pedlar, Martin, Malott, \& Stones, 2000) ou até mesmo ao abuso de idosos (Wolf, 1998).

O estudo comparativo entre cuidadores de três grupos geriátricos diferentes: idosos com DA, com demência vascular e não demenciados, revelou que a sobrecarga do cuidar referida pelos cuidadores é maior naqueles que cuidam de pessoas com DA (Cruz \& Hamdan, 2008).

$\mathrm{Cl}($ esposa/70a), cuidadora de seu marido na fase inicial da doença, e C7(filha/58a), uma das cuidadoras de sua mãe com DA na fase moderada e com amplo apoio e revezamento desse cuidado com a família, não se referiram à sobrecarga do cuidar, queixa frequente entre cuidadores de pessoas com DA, o que demonstra que nessa situação de cuidado o grau da doença não interferiu nessa percepção.

Ao levar em consideração as particularidades da doença de Alzheimer, os cuidadores de pessoas com essa doença desempenham papel fundamental, uma vez que estão envolvidos em praticamente todos os aspectos de cuidados. Eles tendem a assumir um número progressivamente maior de responsabilidades, caminhando da necessidade de ajuda, orientação e auxílio nas atividades mais complexas, como as atividades instrumentais de vida diária (AIVD), para a necessidade de auxílio ou dependência nas atividades de vida diária (AVD), que estão relacionadas ao autocuidado; banho, vestuário, alimentação, higiene e comunicação. C2 (esposa/62a), que cuida sozinha do marido na fase moderada da doença, afirma: "Ele está com dificuldade para tomar banho, trocar de roupa. Está dando muito trabalho. Tem dias que eu não durmo. Só consigo dormir duas a três horas por noite". 
A sobrecarga do cuidar na fase avançada da doença desencadeia o acúmulo de tarefas, o desgaste físico e emocional e o maior comprometimento de recursos financeiros, passando do auxílio a algumas tarefas do paciente para a dependência total nas AVD, que culmina com cuidados no leito.

\section{A doença de várias gerações}

A maior incidência das doenças crônicas, entre elas, a doença de Alzheimer (Small et al., 2007) pode desencadear nos cuidadores maior probabilidade de também desenvolver a doença, como podemos observar em seus depoimentos: C9 (filha/70a) aponta: "Meu pai teve Alzheimer e quem cuidou dele foi minha mãe. Agora minha mãe teve Alzheimer e eu cuidei dela. Então, há possibilidade de eu ter também, não?".

Gonzaga (2012) aponta que pode ocorrer a identificação do cuidador com seu familiar doente, o que pode gerar o medo da repetição da doença em si.

\section{Aspectos positivos do cuidar}

Os efeitos de cuidar para o familiar têm sido bem descritos na literatura, pois produzem mudanças drásticas no estilo de vida, além de representarem abrir mão da própria vida. Por outro lado, estudos recentes têm demonstrado aspectos positivos desse cuidado (Carbonneau, Chantal, \& Desrosiers, 2010; Lloyd, Patterson, \& Muers, 2016; Roth, DilworthAnderson, Huang, Gross, \& Glitin, 2015; Semiatin \& O'Connor, 2012; Shim, Barroso, \& Davis, 2012; Tarlow et al., 2004). Resultados de pesquisas demonstraram que aspectos negativos e positivos relacionados ao cuidar não se apresentam como opostos do mesmo continuum. Numa revisão de literatura, Llyod, Patterson e Muers (2016) apontam que os preditores dos aspectos negativos e positivos da experiência de cuidar em demência não são os mesmos e que variavam em número, sendo que os positivos são sempre em número menor, quando comparados com os negativos. Os autores identificaram oito temas centrais associados aos aspectos positivos de cuidar: (1) satisfação com o papel de cuidar; (2) recompensas emocionais; (3) crescimento pessoal; (4) competência e domínio; (5) fé e crescimento espiritual; (6) ganhos de relacionamento; (7) senso de dever; e (8) reciprocidade.

Os relatos descritos a seguir corroboram os resultados apontados. Em relação às recompensas emocionais, C9 (filha/70a) fala que cuidar da mãe trouxe a possibilidade de ressignificar as experiências vividas antes da doença. Para ela: "O Alzheimer até foi uma coisa boa", pois o longo período em que cuidou de sua mãe a fez receber todo o carinho e afeto não recebidos antes da doença. Vivenciar a experiência diária do cuidar possibilita um novo olhar para a doença que valoriza atos e gestos simples manifestados nas atividades do dia a dia.
C8 (filha/51a) relata "Eu aprendi a não levar a coisa tão a sério, não querer exigir aquilo que a pessoa não consegue mais".

O que pode ser corriqueiro nas relações afetivas torna-se experiência emocionante na demência (Doka, 2004). C7 (filha/58a) passou a valorizar o fazer junto com a mãe, como ir à feirinha de artesanato, comer um doce ou olhar o mar.

Hasselkus e Murray (2007) sugerem que atividades cotidianas com esse novo olhar permitem que a demência possa ser esquecida em alguns momentos e podem trazer satisfação ao cuidador, ao recordar as experiências vividas. C5 (filha/70a) recordou a fala de sua mãe na fase avançada de DA: "A minha mãe, às vezes, à noite, a gente pensava que ela não tinha nenhuma lógica e ela falava: 'Filha, obrigada por tudo que você está fazendo por mim', 'Filha, desculpa o trabalho que eu estou te dando"."

Muitas dessas experiências diárias descritas parecem representar esforços para afastar o estranhamento de viver com a demência. Cuidadores podem desenvolver estratégias de trabalho que colaboram para a manutenção do relacionamento, enquanto fragmentos de normalidade que permanecem nas atividades cotidianas. Essa sensação de reciprocidade entre o cuidador e seu familiar com demência pode ser especialmente significativa (Hasselkus \& Murray, 2007; Lloyd et al., 2016).

A possibilidade de resgatar vínculos rompidos e aproximar-se de pessoas distantes foi identificada em diversas falas. C6 (esposa/65a) descreveu: "Meu filho mora em Minas. Esse ano ele já veio quatro vezes. Eu sei por que ele faz isso. Porque ele tem receio de perder". C2 (esposa/62a), C4 (filha/61a) e C6 (esposa/65a) perceberam a necessidade de cuidar de si para cuidar bem do familiar.

\section{Fase avançada}

A demência pode provocar uma condição de vida limitada, que pode gerar significados de morte associados a esse contexto. Convencionalmente, o morrer é visto como processo e a morte, como evento, mas, em relação às doenças crônicas, entre elas, a demência da doença de Alzheimer, a morte é também um processo (Small et al., 2007).

Nas demências, as pessoas com DA e suas famílias vivem um dilema particular. Não ter clareza que a doença está em fase avançada pode atrasar a preparação psicológica, social e espiritual relacionada com o cuidado para o processo de morrer.

\section{Um caminhar solitário}

Estudo realizado por Hennings, Froggatt e Keady (2010) associou a experiência do cuidar em casos de demência a um território desconhecido, devido à falta de conhecimento da progressão da doença e do processo de 
morrer, e à falha de comunicação com profissionais sobre as opções de tratamento, expondo a pessoa com DA a intervenções com poucos benefícios comprovados, como internação hospitalar, terapia antibiótica e alimentação por sonda.

No contexto das demências, o desafio é a conscientização pública de que se trata de uma doença sem possibilidade de cura, mas que todos os envolvidos podem se beneficiar da abordagem de cuidados paliativos, desde a fase diagnóstica na qual as ações paliativas podem fazer diferença ao longo do processo até a fase final e o pós-óbito (Doka, 2004; Hughes, Jolley, Jordan, \& Sampson, 2007).

C5 (filha/70a) e C9 (filha/70a) exerciam o papel de cuidadores de suas mães com mais de 90 anos de idade na fase avançada da doença. Porém, a forma como suas mães vivenciaram o fim da vida apresentou situações diferentes. C5 descreveu o processo de sua mãe como uma morte dolorosa e sofrida diante de aparelhos, sonda e tratamentos desnecessários em um hospital, enquanto C9 vivenciou o processo final de sua mãe de forma tranquila e em casa.

Em comum, há o fato de que elas não receberam nenhum tipo de orientação sobre os tratamentos adequados no fim de vida e na preparação para o resgate de desejos e preferências de suas mães, já que não tiveram acompanhamento de uma equipe de cuidados paliativos. No Brasil, o Conselho Federal de Medicina (CFM) aprovou a Resolução no 1955, em 2012, a qual dispõe sobre as Diretivas Antecipadas de Vontade (DAV). Porém, no caso das demências são observadas grandes dificuldades legais e bioéticas implicadas na relação médico, paciente e família, quando se trata da tomada de decisões (Burlá, 2015).

O papel dos profissionais de saúde deve estar voltado não apenas para a especificidade do atendimento a essa parcela da população, mas na responsabilidade bioética de ajudar a preservar a identidade dessas pessoas que têm sua autonomia desrespeitada (Burlá, 2015).

Representantes de diversas organizações nacionais norte-americanas reuniram esforços para a formação do grupo de trabalho interdisciplinar intitulado Dementia Measure Work Group (DWG) com o intuito de definir medidas necessárias para profissionais para atingir padrões excelentes de cuidados nas demências, bem como na formação de equipes multi e/ou interdisciplinares (Odenheimer et al., 2013). Nesse contexto, observa-se o esforço internacional para que as ações de cuidado nas demências sejam sistemáticas, abrangentes, consistentes, amplamente aplicadas e de acesso universal.

A WHO (2012) alerta para importância da implantação de cuidados paliativos nas demências o mais cedo possível, juntamente com outros tratamentos para a doença.

\section{Temas transversais a todas as fases e ao pós-óbito}

\section{O luto antecipatório}

Cuidar nas demências pode ser uma das situações mais exigentes que se pode vivenciar, porque, além das questões físicas, financeiras e emocionais, inclui a imprevisibilidade, a longa duração e ambiguidade do processo de adoecimento, que pode levar ao estresse psicológico singular (Fowler, Hansen, Barnato, \& Garand, 2013). Estudos longitudinais têm demonstrado que o luto antecipatório entre os cuidadores familiares de idosos com Alzheimer é um processo dinâmico que oscila entre negação, superenvolvimento, raiva, culpa e aceitação (Holland et al., 2009). C9 (filha/70a) descreve:

Foi muito ruim para mim ver a decadência de minha mãe nessa doença. Eu acho que é a pior coisa que pode acontecer a um ser humano e à família dele, porque é uma coisa angustiante. Você precisa fazer um trabalho de aceitação para você entender o que está acontecendo com aquele ser humano.

Estudo realizado por Paun e Farran (2011) identificou diferenças nas formas de manifestação do luto antecipatório no cuidador familiar, de acordo com o grau de parentesco e com a fase da doença em que o paciente se encontra. $\mathrm{Na}$ fase inicial/moderada os cuidadores/cônjuges apresentam níveis mais baixos de negação da doença, quando comparados aos cuidadores/ filhos. Além disso, os cuidadores/cônjuges lamentavam mais as perdas diárias do que os cuidadores/filhos, principalmente em relação à vida conjugal; como a perda da intimidade, do companheirismo e da amizade. $\mathrm{Na}$ fase avançada, tanto os cuidadores/cônjuges quanto os filhos expressaram maior culpa e pena, com tendência à intensificação desses sentimentos após a morte do familiar.

Viver o desconhecido no dia a dia pode trazer angústia, tristeza, sentimento de impotência, culpa e frustração. C1 (esposa/70a) acompanhou seu marido na fase inicial e relata: "Tem horas que eu fico triste por ver ele assim. A gente fazia tanta coisa juntos".

A identificação de luto antecipatório, mesmo na fase inicial da doença, pode abrir caminho para o desenvolvimento de estratégias de apoio ao bem-estar do cuidador e maximizar sua capacidade de auxiliar a PDA ao longo do processo (Garand et al., 2012).

\section{O luto não reconhecido socialmente}

Esse termo foi utilizado pela primeira por Doka (2004) para ser empregado nos casos em que a pessoa vivencia uma perda que não pode ser admitida abertamente e que não pode ser expressa ou socialmente suportada. No caso das demências, esse luto não é 
reconhecido socialmente porque a realidade do luto vivenciado pela família mantém-se não resolvido devido ao fato de a experiência de perda contínua ser indefinida. Nas demências, o cuidador familiar experimenta a despedida sem a partida, pois o paciente está presente fisicamente, mas psicologicamente ausente, visto que a memória vai gradativamente sendo perdida (Casellato, 2015).

É justamente essa condição de indefinição física ou psicológica que faz com que o luto não seja sancionado ora pelo cuidador familiar, ora por seu contexto social (Casellato, 2015). C2, C4 e C5 não encontraram espaços para desabafar suas dores e sofrimentos no meio familiar ou com os amigos. C4 fala que sente tristeza por não receber visitas enquanto cuida de sua mãe. C5 conta que, quando viu sua mãe no caixão, caiu em prantos e logo foi contida por seu irmão que disse: "Já vai começar?". E completou: "Eu nunca pude liberar meus sentimentos. Eu sempre era reprimida".

Kovács (2003) comenta que os rituais da morte estão se tornando cada vez mais reduzidos atualmente. O processo de luto sofre interferências, pois são valorizadas atitudes mais contidas, como se a dor não existisse.

Doka (2004) aponta que o luto antecipatório pelas perdas causadas pelas síndromes demenciais pode não ser socialmente aceito porque, muitas vezes, a pessoa com demência da doença de Alzheimer é desvalorizada, além de frequentemente ser reconhecida como alguém que já morreu. O luto não reconhecido socialmente ou não compartilhado publicamente pode gerar grande falha no apoio, acolhimento e suporte a essas cuidadoras. Casellato (2015) aponta que o luto não reconhecido acontece a partir do momento em que a sociedade dita "normas" explícitas ou implícitas de quando, por quem, quem, onde e como se enlutar. Dessa forma, ao invés de proporcionar o suporte social necessário aos enlutados, eles passam a ser isolados e sofrem diante de um silêncio tácito em resposta à sua dor e/ou forma de expressá-la.

\section{Considerações finais}

O cuidar em casos de demência tem características que marcam o cotidiano de cuidadores e que podem ser identificadas ao longo do processo de cuidar e no pós-óbito do familiar. As unidades de significados desveladas e agrupadas por fases do processo sugeriram que a fase inicial é marcada por questões relacionadas à dificuldade de acesso ao diagnóstico e ao estigma da doença, as quais impactam os encaminhamentos e direcionamentos às abordagens farmacológicas e não farmacológicas. A fase moderada, caracterizada por maior duração, pode suscitar nesses cuidadores questionamentos que favoreceram a ressignificação de si, da relação com o familiar e da própria experiência de cuidar. Os relatos associados à fase final e após a morte sugeriram que cuidadores familiares experimentaram um processo solitário e doloroso com sofrimento psicológico. Aspectos éticos, legais e biomédicos que são primordiais no cuidado, inclusive nas demências, não se fizeram presentes nos relatos dos entrevistados, o que mostra uma fragilidade na formação de profissionais e no acesso aos recursos e equipamentos que deveriam estar disponíveis desde a fase inicial até o pós-óbito. Outros estudos devem ser conduzidos em diferentes cenários de atenção para melhor compreensão do processo de cuidar em demência.

\section{Alzheimer's disease: the unique experience of family caregivers}

Abstract: Dementia is a slow-progressing chronic syndrome with the subtype of Alzheimer's disease being the most commom (Burlá, 2015). This study verified the experience of care from the perspective of family caregivers of older adults with AD from the qualitative approach. The phenomenological method proposed by Giorgi and Sousa (2010) was adopted. Nine family caregivers (4 wives and 5 daughters) participated during different stages of the disease and after death. From the main question "How is your experience of caring for your relative with Alzheimer's disease?" emerged 13 thematic units associated with each stage of the disease. The findings raise the need of caregivers, which include initial diagnosis and goes through the creation of a space for listening and care, given the gradual loss experienced throughout the caring process. Thus, there must be investments in forming professionals of all fields related to healthcare in order to promote quality of life and well-being for caregivers, as well as the need for interdisciplinary teams for the unique experience of dementia care.

Keywords: dementia, Alzheimer's disease, caregiver family.

\section{La maladie d'Alzheimer : I'expérience unique des aidants familiaux}

Résumé : La démence est un syndrome à évolution lente et progressive, de nature chronique, et son sous-type la maladie d'Alzheimer étant le plus fréquent (Burlá, 2015). De nombreuses études sont réalisées en fonction des demandes du patient, mais du côté de l'aidant familial, elles sont rares. Cette recherche a eu pour objectif connaître l'expérience des soins du point de vue des aidants familiaux des personnes âgées atteints de la MA à travers une abordage qualitative. En ce qui concerne la méthodologie, nous avons choisi l'approche phénoménologique proposée par Giorgi et Sousa (2010). Y ont participé neuf 
membres familiaux (4 épouses et 5 filles) qui ont pris part aux soins à différents stades de la maladie et après le décès de leur proche. De la question centrale : «Comment est l'expérience d'accompagner un membre de sa famille avec la maladie d'Alzheimer ? ", sont émergé 13 thématiques qui ont été associés aux stades de la maladie. Les résultats ont indiqué le besoin signaler par ces aidants de se faire soutenir à partir de l'accès au diagnostic, en ayant un espace de parole que les soient dédié tout au long du processus de l'accompagnement des soins. Ainsi, il s'avère l'importance d'investissement dans la formation de tous les métiers concernant, pour la qualité de vie et le bien-être des aidants et le besoin d'une équipe interdisciplinaire capable de soutenir l'expérience singulière vécue par le membre de la famille qui s'occupe des soins au patient que souffre de démence.

Mots-clés : Démence, Maladie d'Alzheimer, Aidant familial.

\section{La enfermedad de Alzheimer: la experiencia única de los cuidadores familiares}

Resumen: La demencia es un síndrome de curso lento y progresivo, de naturaleza crónica, en la cual se destaca como subtipo más común la enfermedad de Alzheimer -EA- (Burlá, 2015). Se han realizado muchos estudios acerca de las demandas del individuo con demencia, pero son pocos desde la perspectiva del cuidador. A partir de un abordaje cualitativo, la investigación tuvo como objetivo conocer la experiencia del cuidado en la perspectiva de cuidadoras familiares de ancianos con EA. Se utilizó el método fenomenológico propuesto por Giorgi y Sousa (2010). Participaron nueve cuidadoras familiares (4 esposas y 5 hijas). El estudio acompañó el proceso del cuidado en las etapas inicial, intermedia y avanzada, en diferentes fases de la enfermedad y en el postóbito. Desde la cuestión central: "Cómo es para usted la experiencia de acompañar a su familiar con la enfermedad de Alzheimer?", emergieron trece unidades temáticas que se asociaron a las fases de la enfermedad. Los hallazgos apuntan las necesidades de los cuidadores, desde el diagnóstico en la etapa inicial hasta la creación de un espacio para la escucha y el acogimiento en el proceso vivenciado a lo largo del cuidado. Es imprescindible invertir en la formación de profesionales de todas las áreas asociadas al cuidado para promover la calidad de vida y el bienestar de los cuidadores, así como son necesarios equipos interdisciplinarios para la experiencia singular del cuidado en demencia.

Palabras clave: demencia, enfermedad de Alzheimer, cuidador familiar.

\section{Referências}

Alzheimer's Disease International. (2016). World Alzheimer Report 2016: improving health care for people living with dementia. London: Alzheimer's Disease International.

American Psychiatric Association. (2014). Manual diagnóstico e estatístico de transtornos mentais (DSM-5) (5a ed., M. I. C. Nascimento, P. H. Machado, R. M. Garcez, R. Pizzato, S. M. M. Rosa, trads.). Porto Alegre, RS: Artmed.

Barnett, K., Mercer, S., Norbury, M., Watt, G., Wyke, S., \& Guthrie, B. (2012). Epidemiology of multi-morbidity and implication for health care, research and medical education: a cross-sectional study. The Lancet, 380(9836), 37-43. doi: 10.1016/S0140-6736(12)60240-2.

Bédard, M., Pedlar, D., Martin, N. J., Malott, O., \& Stones, M. J. (2000). Burden in caregivers of cognitively impaired older adults living in the community: methodological issues and determinants. International Psychogeriatrics, 12(3), 307-332. Recuperado de https://bit.ly/2SWGN3W

Bell, C. M., Araki, S. S., \& Neumann, P. J. (2001). The association between caregiver burden and caregiver health-related quality of life in Alzheimer disease. Alzheimer Disease and Associated Disorders, 15(3), 129-136.
Brodaty, H., Green, A., Koschera, H., \& Koschera, A. (2003). Meta-analysis of psychosocial interventions for caregivers of people with dementia. Journal American Geriatrics Society, 51(5), 657-664. Recuperado de https://bit.ly/2v1s4fZ

Brodaty, H., Gresham, M., \& Luscombe, G. (1997). The Prince Henry Hospital dementia caregiver's training programme. International Journal of Geriatric Psychiatry, 12(2), 183-192. doi: 10.1002/(SICI)10991166(199702)12:2<183::aid-gps584>3.0.co;2-j

Burlá, C. (2015). A aplicação das diretivas antecipadas de vontade na pessoa com demência (Tese de doutorado). Recuperado de https://bit.ly/3bUukGr

Carbonneau, H., Chantal, C., \& Desrosiers, J. (2010). Development of a conceptual framework of positive aspects of caregiving in dementia. Dementia, 9(3), 327-353. doi: $10.1177 / 1471301210375316$

Casellato, G. (2015). O resgate da empatia: suporte psicológico ao luto não reconhecido. In G. Casellato (Org.), Luto não reconhecido: o fracasso da empatia nos tempos modernos (pp. 15-28). São Paulo, SP: Summus.

Conselho Federal de Medicina. (2012). Resolução CFM $n^{o} 1.995 / 2012$, de 31 de agosto de 2012. Recuperado de https://bit.ly/3bS6mvv 
Cruz, M. N., \& Hamdan, A. C. (2008). O impacto da doença de Alzheimer no cuidador. Psicologia em Estudo, 13(2), 223-229. Recuperado de https://bit.ly/37MaFFr

Dizazzo-Miller, R., Samuel, P. S., Barnas, J. M., \& Welker, K. M. (2014). Addressing everyday challenges: feasibility of a family caregiver training program for people with dementia. The American Journal of Occupational Therapy, 68, 212-220. doi: 10.5014/ajot.2014.009829

Doka, K. J. (2004). Grief and dementia. In K. J. Doka (Ed.), Living with grief: Alzheimer's Disease (pp. 140-151). Washington, DC: Hospice Foundation of America.

Eloniemi-Sulkava, E., Saarenheimo, M., Laakkonen, M. L., Pietilä, M., Savikko, N., Kautiainen, H. ... Pitkälä, K. H. (2009). Family care as collaboration: effectiveness of a multicomponent support program for elderly couples with dementia: randomized controlled intervention study. Journal of the American Geriatrics Society, 57(12), 2200-2208. doi: 10.1111/j.1532-5415.2009.02564.x

Farran, C. J., Keane-Hagerty, E., Salloway, S., Kupferer, S., \& Wilken, C. S. (1991). Finding meaning: an alternative paradigm for Alzheimer's disease family caregivers. Journal of Gerontology, 31(4), 483-489.

Fonseca, J. P. (2004). Luto antecipatório: as experiencias pessoais, familiares e sociais diante de uma morte anunciada. Campinas, SP: Livro Pleno.

Fowler, N. R., Hansen, A. S., Barnato, A. E., \& Garand, L. (2013). Association between anticipatory grief and problem solving among family caregivers of persons with cognitive impairment. Journal of Aging and Health, 25(3), 493-509. doi: 10.1177/0898264313477133

Frank, C. (2017). A patient's experience in dementia care: using the "lived experience" to improve care. Canadian Family Physician Le Médicin de Famille Canadien, 63, 22-26. Recuperado de https://bit.ly/2HCX60m

Gallagher-Thompson, D., \& Powers, D. V. (1997). Primary stressors and depressive symptoms in caregivers of dementia patients. Aging and Mental Health, 1(3), 248-255. doi: 10.1080/13607869757146

Garand, L., Lingler, J. H., Deardorf, K. E., DeKosky, S. T., Schulz, R., Reynolds, C. F. ... Dew, M. A. (2012). Anticipatory grief in new family caregivers of persons with mild cognitive impairment and dementia. Alzheimer Disease and Association Disorders, 26(2), 159-165. doi: 10.1097/WAD.0b013e31822f9051

Giorgi, A., \& Sousa, D. (2010). Método fenomenológico de investigação em Psicologia. Lisboa: Fim de Século.

Gonzaga, L. Z. M. (2012). Morte anunciada: implicações para o luto dos cuidadores familiares (Dissertação de mestrado). Recuperado de https://bit.ly/2SMMFOm

Haley, W. E., Bergman, E. J., Roth, D. L., McVie, T., Gaugler, J. E., \& Mittelman, M. S. (2008). Long-term effects of bereavement and caregiver intervention on dementia caregiver depressive symptoms. The Gerontologist, 48(6), 732-740. Recuperado de https://bit.ly/2 VanIOu

Hasselkus, B. R., \& Murray, B. J. (2007). Everyday occupation, well-being, and identify: the experience of caregivers in families with dementia. The American
Journal of Occupational Therapy, 61(1), 9-20. doi: 10.5014/ajot.61.1.9

Hennings, J., Froggatt, K., \& Keady, J. (2010). Approaching the end of life and dying with dementia in care homes: the accounts of family carers. Reviews in Clinical Gerontology, 20(2), 114-127. doi:10.1017/S0959259810000092

Holland, J. M., Currier, J. M., \& Gallagher-Thompson, D. (2009). Outcomes from the resources for enhancing Alzheimer's caregiver health (REACH) program for bereaved caregivers. Psychology and Aging, 24(1), 190-202. doi: 10.1037/a0014303

Holley, C. (2009). Anticipatory grief in the context of dementia caregiving (Tese de doutorado). Recuperado de https://bit.ly/2SMNYge

Hughes, J. C., Jolley, D., Jordan, A., \& Sampson, E. L. (2007). Palliative care in dementia: issues and evidence. Advances in Psychiatric Treatment, 13(4), 251-260. doi: 10.1192/apt.bp.106.003442

Instituto de Pesquisa Econômica Aplicada. (2011). Infraestrutura social e urbana no Brasil: subsidios para uma agenda de pesquisa e formulação de políticas públicas. Recuperado de https://bit.ly/39Ob0c4

Jamieson, M., Grealish, L., Brown, J.-A., Draper, B. (2016). Carers: the navigators of maze of care for people with dementia: a qualitative study. Dementia, 15(5), 1112-1123. doi: 10.1177/1471301214554930

Kovács, M. J. (2003). Educação para a morte: temas e reflexões. São Paulo, SP: Casa do Psicólogo.

Kovács, M. J. (2005). Educação para a morte. Psicologia Ciência e Profissão, 25(3), 484-497. doi: 10.1590/S1414-98932005000300012

Lloyd, J., Patterson, T., \& Muers, J. (2016). The positive aspects of caregiving of dementia: a critical review of the qualitative literature. Dementia, 15(6), 1534-1561. doi: 10.1177/1471301214564792

Morais, S. R. S. (2011). Sob a espada de Dâmocles: a prática de psicólogas em oncologia pediátrica no município de Pernambuco-RE (Tese de Doutorado). Recuperado de https://bit.ly/3bX2pWe

Odenheimer, G., Borson, S., Sanders, A. E., Swain-Eng, R. J., Kyomen, H. H., Tierney, S. ... Johnson, J. (2013). Quality improvement in neurology: dementia management quality measures (Executive Summary). The American Journal of Occupational Therapy, 67(6), 704-710. doi:10.5014/ajot.2013.676004

Paun, O., \& Farran, C. J. (2011). Chronic grief management for dementia caregivers in transition: intervention development and implementation. Journal of Gerontological Nursing, 37(12), 28-35. doi: 10.3928/00989134-20111103-04

Prorok, J. C., Horgan, S., \& Seitz, D. P. (2013). Health care experiences of people with dementia and their caregivers: a meta-ethnographic analysis of qualitative studies. Canadian Medical Association Journal, 185(14), 669-680. doi: 10.1503/cmaj.121795 
Raivio, M. M., Laakkonen, M.-L., \& Pitkälä, K. H. (2011). Alzheimer's patients spouse critiques of the support services. ISRN Nursing, 1-29. doi:10.5402/2011/943059

Rando, T. A. (1986). A comprehensive analysis of anticipatory grief: perspectives, processes, promises, and problems. In T. A. Rando (Ed.), Loss and anticipatory grief (pp. 1-36). New York: Lexington Books.

Rolland, J. S. (1998). Ajudando as famílias com perdas antecipadas. In F. Walsh \& M. McGoldrick (Orgs.). Morte na familia: sobrevivendo às perdas (pp. 166-186). Porto Alegre, RS: Artmed.

Roth, D. L., Dilworth-Anderson, P., Huang, J., Gross, A. L., \& Glitin, L. N. (2015). Positive aspects of family caregivers for dementia: differential items functioning by race. Journals of Gerontology: Psychological Sciences, 70(6), 813-819. doi: 10.1093/geronb/gbv034

Sanders, S., \& Corley, C. S. (2003). Are they grieving? A qualitative analysis examining grief in caregivers of individuals with Alzheimer's disease. Social Work in Health Care, 37(3), 35-53. doi: 10.1300/J010v37n03_03

Semiatin, A., \& O'Connor, M. K. (2012). The relationship between self-efficacy and positive aspects of caregiving in Alzheimer's disease caregivers. Aging and Mental Health, 16(6), 683-688. doi: 10.1080/13607863.2011.651437

Shim, B., Barroso, J., \& Davis, L. L. (2012). A comparative qualitative analysis of stories of spousal caregivers of people with dementia: negative, ambivalent, and positive experiences. International Journal of Nursing Studies, 49(2), 220-229. doi: 10.1016/j.ijnurstu.2011.09.003

Silva, D. J. R. (2012). O cuidado e a velhice-dependente: considerações antropológicas sobre a doença de Alzheimer (Dissertação de mestrado). Recuperado de https://bit.ly/37J0W2D

Small, N., Froggatt, K., \& Downs, M. (2007). Living and dying with dementia: dialogues about palliatives care. Oxford: Oxford University Press.

Tarlow, B. J., Wisniewsky, S. R., Belle, S. H., Rubert, M., Ory, M. G., \& Gallagher-Thompson, D. (2004). Positive aspects of caregiving: contributions of the REACH Project to the development of new measures for Alzheimer's caregiving. Research on Aging, 26(4), 429-453. doi: 10.1177/0164027504264493

Wolf, R. S. (1998). Caregiver stress, Alzheimer's disease, and elder abuse. American Journal of Alzheimer's Disease, 13, 81-83. Recuperado de https://bit.ly/2wzkfP8

World Health Organization. (2012). Dementia: a public health priority. Geneva: WHO.

Recebido: 02/02/2018

Revisado: 01/03/2019

Aprovado: 20/11/2019 